\title{
Upwelling and Coastal Jets in a Continuously Stratified Ocean
}

\author{
J. S. Allen \\ Dept. of Aerospace Engineering, The Pennsylvania State University, University Park 16802
}

(Manuscript received 26 December 1972, in revised form 12 February 1973)

\begin{abstract}
A simple, linear, two-dimensional, $f$-plane model of coastal upwelling in a continuously stratified ocean is investigated. The transient response of the fluid to an impulsively applied alongshore wind stress, the nature of the approach to a steady state, and the final steady state flow are studied. Particular attention is given here to an examination, in the continuously stratified case, of the interrelation of upwelling and the phenomenon of a baroclinic coastal jet. The coastal jet is a feature which has appeared in studies of the transient response of two-layer fluid models near a coast. The stratification parameter $S=(\delta N / f)^{2}$, where $\delta=I I / L$ is the aspect ratio, $H$ the depth, $L$ a characteristic horizontal scale, $N$ the Brunt-Väisälä frequency, and $f$ the Coriolis parameter, is assumed to lie in the range $\mathrm{O}\left(E_{V}{ }^{3}\right)<S \lambda \leqslant \mathrm{O}(1)$, where $\lambda=E_{V} / E_{H}$ and where $E_{H}$ and $E_{V}$ are respectively the horizontal and vertical Ekman numbers. It is also assumed that $L$ is large enough that $S \ll 1$ and $\lambda \gg 1$. These are the conditions for which, during the transient flow, a baroclinic coastal jet is present in an inviscid upwelling layer of width $\mathrm{O}\left(S^{\frac{1}{2}}\right)$. It also appears that these conditions are reasonable for oceanic upwelling regions. The jet development is initiated on a time scale of $\mathrm{O}\left(E_{V}{ }^{-\frac{1}{2}}\right)$. A steady state is reached in the $O\left(S^{1}\right)$ upwelling layer on a longer diffusive time scale of $\mathrm{O}\left(S E_{H}{ }^{-1}\right)$. For $S \lambda<\mathrm{O}(1)$, the alongshore current outside the $\mathrm{O}\left(S^{\frac{1}{3}}\right)$ layer increases in magnitude with time, by diffusive spreading of the boundary effects, in an additional boundary layer of width $\mathrm{O}\left(\lambda^{-\frac{1}{2}}\right)$. It reaches a steady state on a yet longer time scale of $\mathrm{O}\left(E_{V}{ }^{-1}\right)$. For $S \lambda=\mathrm{O}(1)$, the two boundary layers merge and adjust on the same time scale. The final steady state is characterized by a coastal current which is confined to the $\mathrm{O}\left(S^{\frac{1}{2}}\right)$ and $\mathrm{O}\left(\lambda^{-\frac{1}{3}}\right)$ boundary layers.
\end{abstract}

\section{Introduction}

A simple model of coastal upwelling in a continuously stratified ocean is investigated. The Coriolis parameter is assumed to be a constant ( $f$-plane) and the flow is assumed to be independent of the alongshore direction and, therefore, to be two-dimensional, with the two spatial dimensions lying in a plane normal to the coast. An idealized geometry is utilized. The depth is constant and the coast is represented by a vertical wall. An alongshore, surface wind stress is applied impulsively to the fluid, which is assumed to be initially at rest. The stress is nonzero at the coast and can have a general variation in the offshore direction; it remains constant in time after the initial impulsive application. The model is linearized in the usual manner by the assumption that the motion results in negligible nonlinear flind accelerations and in small departures from an equilibrium, linear density distribution. The density is assumed to depend linearly on the temperature and salinity and an apparent temperature (Fofonoff, 1962) is utilized. Turbulent exchange processes are represented by constant eddy coefficients. The transient response of the fluid, the nature of the approach to a steady state, and the final steady state flow are studied.

The impulsive application of a constant (in time) alongshore wind stress is supposed to model, in a very idealized manner, the initiation and maintenance of an upwelling season by the onset and continuation of upwelling-favorable mean winds. Since the problem studied is linear, the solutions can also be thought of as describing the response of the fluid during the upwelling season to a sudden change in the wind field, where, for example, relatively weak winds, over a period of several days, suddenly increase in magnitude and remain strong for a subsequent time period of several days.

Primary attention is given here to a study of upwelling and its interrelation with the phenomenon of a coastal jet. The coastal jet is a feature that has emerged from studies of the transient response of two-layer fluid models near a coast (Charney, 1955; Csanady, 1968; O'Brien and Hurlburt, 1972). This baroclinic jet appears adjacent to the coast during the initial stages of the response to an impulsively applied wind stress with an alongshore component. It is confined to a region of dimensionless width $O\left(F^{-\frac{1}{2}}\right)$, where $F^{-\frac{1}{2}}=\bar{\delta} \bar{N} / f, \bar{\delta}=D / L$, $\bar{N}^{2}=\frac{1}{2} g \Delta \rho /\left(\rho_{2} D\right), D=2 H_{1} H_{2} /\left(H_{1}+H_{2}\right)$, and $\Delta \rho=\rho_{2}-\rho_{1}$. Here $\rho_{1}, \rho_{2}$ and $H_{1}, H_{2}$ are the densities and depths of the upper and lower layers, respectively, $g$ is the acceleration of gravity, $L$ a characteristic horizontal scale, and $f$ the Coriolis parameter. The jet occurs in a thin boundary layer for $F^{-\frac{1}{2}} \ll 1$. An analogous phenomenon should be expected to occur in a continuously stratified fluid and, since a relatively strong alongshore current is observed in upwelling regions (Mooers et al., 1973), its characteristics should be investigated.

From the results of the two-layer studies, we should expect the coastal jet to exist in a boundary layer when 
the stratification parameter $S=(\delta N / f)^{2}$, where $\delta=H / L$ is the aspect ratio, $H$ the depth, and $N$ the BruntVäisälä frequency, is small. We may also anticipate that the dimensionless width of the jet will have a scale $\mathrm{O}\left(S^{\frac{1}{2}}\right)$. In fact, in a recent paper, Walin (1972) has studied the inviscid, hydrographic response of a confined, continuously stratified fluid, like the Baltic, to transient disturbances. He finds a barotropic response in the interior and a baroclinic response in a narrow coastal region such as the above. Related results of a similar nature were found by Allen (1971). The present study is related to Walin's. However, here, with a more idealized model, the objective is to obtain a description of the upwelling circulation and to study its relationship with a coastal current. In addition, an initial-value (spin-up) problem is treated and the role of diffusive effects in limiting the initial, inviscid, baroclinic response is studied.

We consider the stratification parameter $S$ to lie in the range $\mathrm{O}\left(E_{V^{\frac{1}{2}}}\right)<S \lambda<\mathrm{O}(1)$, where $\lambda=E_{V} / E_{H}$ and where $E_{H}$ and $E_{V}$ are respectively the horizontal and vertical Ekman numbers. We also assume that the characteristic horizontal scale $L$ is large enough that $S \ll 1$ and $\lambda \gg 1$. As we will show, these are the conditions for which, during the transient flow, a baroclinic coastal jet is present in an inviscid upwelling boundary layer of thickness $\mathrm{O}\left(S^{\frac{1}{2}}\right)$. The jet is characterized by relatively large alongshore currents. The jet development is initiated on a time scale of $O\left(E_{V}{ }^{-\frac{1}{2}}\right)$ in which the upwelling circulation and one component of the alongshore flow reach a quasi-steady state. The additional component of the alongshore flow, which initially comprises the coastal jet, reaches a steady state in the $O\left(S^{\frac{1}{2}}\right)$ upwelling layer on a longer diffusive time scale of $O\left(S E_{H}^{-1}\right)$. For $S \lambda<O(1)$, the alongshore current outside the $O\left(S^{\frac{1}{2}}\right)$ layer increases in magnitude with time, by diffusive spreading of the boundary effects, in an additional boundary layer of width $O\left(\lambda^{-\frac{1}{2}}\right)$. It reaches a steady state of a yet longer time scale of $\mathrm{O}\left(E_{V}{ }^{-1}\right)$. For $S \lambda=\mathrm{O}(1)$, the two boundary layers merge and adjust on the same time scale. In the final steady state, there is a coastal current which is confined to the $O\left(S^{\frac{1}{2}}\right)$ and $O\left(\lambda^{-\frac{1}{2}}\right)$ boundary layers.

It appears that the ranges of parameter values that are appropriate for the appearance of a coastal jet in this idealized model are reasonable for oceanic coastal upwelling regions, although uncertainties in the values of the appropriate eddy coefficients make the parameter range estimates uncertain. The applicability of the results to the oceanic upwelling region off the coast of Oregon is discussed in Section 5.

\section{Formulation}

We consider an incompressible fluid which satisfies the Boussinesq approximation and which is situated on an $f$-plane which effectively rotates with uniform angular velocity $\boldsymbol{\Omega}=\Omega \hat{\mathbf{k}}=\frac{1}{2} f \hat{\mathbf{k}}$, where $f$ is the (constant)
Coriolis parameter and $\hat{\mathbf{k}}$ a constant unit vector in the $z$ (vertical) direction in a Cartesian $(x, y, z)$ coordinate system. An apparent temperature (Fofonofi, 1962) is utilized and constant eddy coefficients are used to represent the turbulent exchange processes. The governing equations are

$$
\begin{aligned}
\nabla \cdot \mathbf{q} & =0, \\
\mathbf{q}_{t}+\mathbf{q} \cdot \nabla \mathbf{q}+2 \mathbf{\Omega} \times \mathbf{q} & =-\left(1 / \rho_{0}\right) \nabla p-\left(\rho / \rho_{0}\right) g \hat{\mathbf{k}} \\
+A_{H} \nabla_{H}{ }^{2} \mathbf{q}+A_{V} \mathbf{q}_{z z}, & \\
T_{t}+\mathbf{q} \cdot \nabla T & =K_{H} \nabla_{H}{ }^{2} T+K_{V} T_{z z} \\
\rho & =\rho_{0}\left[1-\alpha\left(T-T_{0}\right)\right]
\end{aligned}
$$

where the subscripts $t$ and $z$ denote partial differentiation and where $\nabla_{H}{ }^{2}=\partial^{2} / \partial x^{2}+\partial^{2} / \partial y^{2}$. The variables q, $p, \rho$ and $T$ are respectively the velocity, pressure, density and (apparent) temperature of the fluid; $\rho_{0}$ and $T_{0}$ are constant reference values of the density and temperature; $\alpha$ is the effective coefficient of thermal expansion; and $A_{H}, A_{V}$ and $K_{I I}, K_{V}$ are the constant horizontal and vertical eddy coefficients for momentum and temperature.

We consider a linear equilibrium temperature and density distribution

$$
T_{s}=T_{0}+\Delta T_{0} z / H, \quad \rho_{s}=\rho_{0}\left(1-\alpha \Delta T_{0} z / H\right),
$$

where $\Delta T_{0}(>0)$ is the basic temperature difference imposed over the depth $\mathbf{H}$.

The variables are nondimensionalized in the following manner, where $(u, v, w)$ are the velocity component in the $x, y, z$ directions:

$$
\left.\begin{array}{l}
x=L x^{*}, \quad y=L y^{*}, \quad z=H z^{*}, \quad t=\Omega^{-1} l^{*} \\
u=U u^{*}, \quad v=U v^{*}, \quad w=(H / L) U w^{*} \\
\begin{array}{l}
p=p_{0}-\rho_{0} g H z^{*}+\frac{1}{2} \rho_{0} g H \alpha \Delta T_{0} z^{* 2}+\rho_{0} U \Omega L p^{*} \\
T=T_{s}+(\Omega U L / \alpha g H) T^{*}, \quad \rho=\rho_{s}+\left(\rho_{0} U \Omega L / g H\right) \rho^{*}
\end{array}
\end{array}\right\},
$$

where $L$ is a characteristic horizontal scale, $U$ a characteristic horizontal velocity, and $p_{0}$ a constant reference pressure. The motion is driven by an applied stress $\tau$ which we nondimensionalize, $\tau=\tau_{0} \tau_{T}{ }^{*}$, with $\tau_{0}$ which is the magnitude of the applied stress at the coast. We then assume that the characteristic velocity $U=H \tau_{0} /\left(\rho_{0} A_{V}\right)$.

The coordinate system is placed with the $y$-axis aligned in the alongshore direction. For two-dimensional flow $(\partial / \partial y \equiv 0)$, the dimensionless equations are (dropping the asterisks)

$$
\begin{aligned}
u_{x}+w_{z} & =0, \\
u_{t}+\epsilon \mathbf{q} \cdot \nabla u-2 v & =-p_{x}+E_{H} u_{x x}+E_{V} u_{z z}, \\
v_{t}+\epsilon \mathbf{q} \cdot \nabla v+2 u= & E_{I} v_{x x}+E_{V} v_{z z}, \\
\delta^{2} w_{t}+\delta^{2} \epsilon \mathbf{q} \cdot \nabla w= & -p_{z}+T \\
& +\delta^{2}\left(E_{H} w_{x x}+E_{V} w_{z z}\right), \\
T_{t}+\epsilon \mathbf{q} \cdot \nabla T+4 S w= & \left(E_{I I} / \sigma_{H}\right) T_{x x}+\left(E_{V} / \sigma_{V}\right) T_{z z},
\end{aligned}
$$

where $\epsilon=U /(\Omega L)$ is the Rossby number, $\delta=H / L$ is the 
aspect ratio, $V^{2}=g \alpha \Delta T_{0} / H$ is the square of the BruntVäisälä frequency, $S=(\delta N / 2 \Omega)^{2}$ is the stratification parameter, $E_{H}=A_{H} / \Omega L^{2}$ and $E_{V}=A_{V} / \Omega H^{2}$ are respectively the horizontal and vertical Ekman numbers, and $\sigma_{I I}=A_{H} / K_{H}$ and $\sigma_{V}=A_{V} / K_{V}$ are respectively the horizontal and vertical turbulent Prandtl numbers.

We consider cases where $\delta \ll 1$ and where the Rossby number $\epsilon$ is small enough that the nonlinear terms multiplied by $\epsilon$ can be neglected. The resulting equations are

$$
\begin{aligned}
u_{x}+w_{z} & =0, \\
u_{t}-2 v & =-p_{x}+E_{H I} u_{x x}+E_{V} u_{z z}, \\
v_{t}+2 u & =E_{I} v_{x x}+E_{V} v_{z z}, \\
0 & =-p_{z}+T, \\
T_{t}+4 S w & =\left(E_{H} / \sigma_{H}\right) T_{x x}+\left(E_{V} / \sigma_{V}\right) T_{z z} .
\end{aligned}
$$

The fluid is contained in a rectangular region of a constant depth $H$ and length $L$, i.e., within the region $0 \leqslant x \leqslant 1,0 \leqslant z \leqslant 1$ in dimensionless coordinates. The bottom is at $z=0$; the top surface is at $z=1$ and is bounded by a rigid lid. The vertical wall at $x=1$ represents the coast of interest. There is no heat transfer at the coast, i.e., $T_{x}(x=1)=0$. The fluid is confined by an additional vertical wall at $x=0$. A wall is placed at $x=0$ strictly for convenience in the problem formulation; it does not affect the upwelling process near the coast at $x=1$. The fluid is at rest initially and at $t=0 \mathrm{a}$ surface stress $\tau=\tau_{T}(x) \hat{\mathbf{j}}$ is applied impulsively. The distribution of $\tau_{T}(x)$ is such that

$$
\tau_{T}(x=1)=-1,
$$

to produce upwelling at the coast $x=1$, and

$$
\tau_{T}(x=0)=0
$$

to simplify the analysis and avoid boundary layers at $x=0$. The temperature boundary conditions, at the top and bottom surface, are taken for simplicity to be that of a fixed constant temperature (zero perturbation temperature) at the top surface and a fixed constant heat flux (zero perturbation temperature heat flux) at the bottom surface.

The resultant initial and boundary conditions for the problem are as follows:

$$
\begin{array}{ll}
u=v=w=T=0, & \text { at } t=0, \\
u=v=0, \quad T_{x}=0, & \text { at } x=0,1, \\
u=v=w=0, \quad T_{z}=0, & \text { at } z=0, \\
w=u_{z}=0, \quad v_{z}=\tau_{T}, \quad T=0, & \text { at } z=1(t>0) .
\end{array}
$$

We assume that the vertical Ekman number is small

that

$$
E_{V} \ll O(1),
$$

and that

$$
\sigma_{V}=\sigma_{H}=\sigma=\mathrm{O}(1),
$$

$$
\mathrm{O}\left(E_{V^{\frac{1}{3}}}\right)<S \lambda \leqslant \mathrm{O}(1)
$$

where $\lambda=E_{V} / E_{I I}$. The assumption that the Prandtl numbers are equal $[(2.5 \mathrm{~b})]$ is made for convenience in obtaining a solution in Section 4 . Note that $(2.5 \mathrm{a}, \mathrm{c})$ do not involve the artificial horizontal scale $\mathrm{L}$. We assume, however, that $L$ is large enough that

$$
S \ll 1, \quad \lambda \gg 1 .
$$

Boundary layer methods will be used for the limits $E_{V}, S \rightarrow 0$, subject to (2.5).

After the initial application of the stress, a thin Ekman layer of thickness $\mathrm{O}\left(E_{V}\right)$ will form at the top surface on a short, $O(1)$ time scale. We follow the usual simplifying assumption for spin-up problems (Greenspan, 1968) and neglect the time development of the flow in the Ekman layer, by treating it as quasisteady. As a result the condition (e.g., Pedlosky, 1968) for the vertical component of velocity of the interior flow at the top surface is

$$
w(x, z=1)=\frac{1}{2} E_{\mathrm{V}} \tau_{T x} .
$$

The Ekman layer at the bottom surface is also treated as quasi-steady and the resultant condition there (Greenspan, 1968) is

$$
w(x, z=0)=\frac{1}{2} E_{Y^{\frac{1}{2}} v_{x}}(x, z=0) .
$$

Conditions (2.6) are not valid, of course, in small corner regions near the coast.

\section{The initial spin-up : $t=\mathrm{O}\left(E_{V}^{-\frac{1}{2}}\right)$}

The boundary conditions (2.6) dictate that the interior flow, away from boundary layers, will develop initially on the spin-up time scale for homogeneous fluids, $t=\mathrm{O}\left(E_{V^{-\frac{1}{2}}}\right)$ [Holton, 1965]. For the moment, we ignore the fact that $S$ is a small parameter and we treat $S$ as if it were $O(1)$. The variables are scaled in the following manner:

$$
\left.\begin{array}{rl}
t & =E_{V}{ }^{-\frac{1}{2}} \bar{t} \\
u & =E_{V} u_{0}(x, z, \bar{l})+\cdots, \quad y=E_{V}{ }^{\frac{1}{2}} v_{0}+\cdots, w=E_{V} w_{0}+\cdots \\
p & =E_{V^{\frac{1}{2}}} p_{0}+\cdots, \quad T=E_{V}^{\frac{1}{2}} T_{0}+\cdots
\end{array}\right\}
$$

The resulting equations are

$$
\begin{gathered}
u_{0 x}+w_{0 z}=0, \\
2 v_{0}=p_{0 x}, \quad v_{0 \tilde{t}}+2 u_{0}=0, \\
T_{0}=p_{0 z}, \quad T_{0 \tilde{t}}+4 S_{w \mho_{0}}=0 .
\end{gathered}
$$

It follows from (3.2) that the potential vorticity is time-independent, i.e.,

$$
\left[v_{0 x}+(2 S)^{-1} T_{0 z}\right]_{\bar{t}}=0 .
$$

From (3.3), one equation for the pressure may be obtained :

$$
\left(p_{0 x x}+S^{-1} p_{0 z z}\right)_{i}=0 .
$$

Rather than work with (3.4), however, it is convenient to introduce a streamfunction (Siegmann, 1971) such 
that

$$
u_{0}=\psi_{0 z}, \quad w_{0}=-\psi_{0 x}
$$

i.e., $\psi=E_{V} \psi_{0}+\cdots$.

The equation for $\psi_{0}$, from (3.2), is

$$
\nabla_{S}^{2} \psi_{0}=\psi_{0 x x}+S^{-1} \psi_{0 z z}=0
$$

Boundary conditions on $\psi_{0}$, from (2.4) and (2.6) are

$$
\begin{gathered}
\psi_{0}(z=1)=-\frac{1}{2} \tau_{T}, \quad \psi_{0 i}(z=0)=\psi_{0 z}(z=0), \\
\psi_{0}(x=0,1)=0 .
\end{gathered}
$$

Note that these conditions on $\psi_{0}$ require a concentrated inflow into the surface Ekman layer at the coast, $x=1$. This inflow will be through two corner regions, which are discussed in the Appendix, and will be reflected as a singularity in the derivatives of $\psi_{0}$ at $x=1, z=1$.

The required initial conditions are

$$
\nabla_{S}{ }^{2} \psi_{0}(\bar{l}=0)=0, \quad \psi_{0}(z=0, \bar{t}=0)=0 .
$$

The solution for $\psi_{0}$ may be obtained with the use of the Laplace transform. Representing $\tau_{T}$ as

$$
\tau_{T}=\sum_{n=1}^{\infty} \tau_{T n} \sin n \pi x
$$

we find

$$
\begin{aligned}
\psi_{0}=-\frac{1}{2} \sum_{n=1}^{\infty} \tau_{T n}\left\{\frac{\sinh \alpha_{n} z}{\sinh \alpha_{n}} \exp \left(-\lambda_{n} \bar{t}\right)\right. \\
\left.+\frac{\cosh \alpha_{n} z}{\cosh \alpha_{n}}\left[1-\exp \left(-\lambda_{n} \bar{t}\right)\right]\right\} \sin n \pi x,
\end{aligned}
$$

where $\alpha_{n}=n \pi S^{x}$ and $\lambda_{n}=\alpha_{n} \operatorname{coth} \alpha_{n}$. Expressions for $v_{0}$ or $T_{0}$ may be obtained from the time integration of (3.2c,e) using $v_{0}(\dot{t}=0)=T_{0}(\dot{t}=0)=0$. [Note that $\psi_{0}(\bar{t}=0) \neq 0$. It corresponds to the flow field present after the initial development of the surface Ekman layer.] The resulting expression for $v_{0}$ is

$$
\begin{aligned}
v_{0}= & \sum_{n=1}^{\infty} \tau_{T n} \alpha_{n}\left\{\frac{\cosh \alpha_{n} z}{\sinh \alpha_{n}} \lambda_{n}{ }^{-1}\left[1-\exp \left(-\lambda_{n} \bar{t}\right)\right]\right. \\
& \left.+\frac{\sinh \alpha_{n} z}{\cosh \alpha_{n}}\left\{\bar{t}-\lambda_{n}{ }^{-1}\left[1-\exp \left(-\lambda_{n} \bar{t}\right)\right]\right\}\right\} \sin n \pi x .
\end{aligned}
$$

Eqs. (3.10) (and a corresponding expression for $T_{0}$ ) give a complete solution for the inviscid interior flow on the $\bar{t}$ time scale and are similar in form to results obtained previously by other investigators (e.g., Holton, 1965). As $t \rightarrow \infty, \psi_{0}$ reaches a quasi-steady state, while $v_{0}$ has one component that reaches a quasi-steady state and an additional component that is increasing linearly in $\bar{t}$, corresponding to the driving by a quasisteady $u_{0}$ in (3.2c). This form of the solution, although holding for small values of $S$ [i.e., as we will see, for $\left.S>\mathrm{O}\left(E_{H} E_{V}-1\right)\right]$, is not very useful for a physical interpretation of the behavior of the flow for $S \ll 1$. For that purpose, it is better to consider a perturbation analysis for small values of $S$. We may note from (3.6), however, that for $S \ll 1$ a boundary layer with scale $\delta_{x}=\mathrm{O}\left(S^{\frac{1}{2}}\right)$ is indicated if both terms are to balance.

To proceed with a perturbation analysis for $S \ll 1$, we expand the interior variables in the same manner as in (3.1), with the exception that $T=S E_{V^{\frac{1}{2}}} T_{0}+\cdots$. The first three equations are the same as $(3.2 \mathrm{a}, \mathrm{b}, \mathrm{c})$. Eq. (3.2d) becomes

$$
p_{0 z}=0 \text {, }
$$

which uncouples (3.2e) from (3.2a,b,c) and (3.11). The resulting problem is the same as for a homogeneous fluid. In particular, it follows that

The solution is

$$
v_{0 z}=0, \quad w_{0 z z}=0 \text {. }
$$

$$
\begin{aligned}
\psi_{0} & =\frac{1}{2} \tau_{T}[(1-z) \exp (-\bar{t})-1], \\
\vartheta_{0} & =\tau_{T}[1-\exp (-\bar{t})] .
\end{aligned}
$$

A boundary layer is necessary at $x=1$ since, with $\tau_{T}(x=1) \neq 0,(3.13 \mathrm{a})$ does not satisfy (3.7c). Note that with $\tau_{T}(x=0)=0$, a similar type of boundary layer is not needed at $x=0$. Since it is indicated by (3.6), we assume that the boundary layer has a width $\delta_{x}=\mathrm{O}\left(S^{\frac{1}{2}}\right)$ and we define

$$
\xi=S^{-\frac{1}{2}}(1-x) .
$$

For the moment, we keep in $(2.2 \mathrm{c}, \mathrm{e})$ the largest viscous and heat conduction terms, $v_{x x}$ and $T_{x x}$. One equation can be derived from (2.2) for the pressure in the boundary layer, i.e.,

$$
\left(p_{\xi \xi}+p_{z z}\right)_{i}=\left(E_{V^{\frac{1}{2}}} / S \lambda\right)\left(p_{\xi \xi}+\sigma^{-1} p_{z z}\right)_{\xi \xi} .
$$

We can see from (3.14) that, if $S \lambda=\mathrm{O}\left(E_{V^{\frac{1}{2}}}\right)$, the viscous and heat conduction terms are important in an $\mathrm{O}\left(S^{\frac{1}{2}}\right)$ boundary layer on the $\bar{t}$ time scale. If, however, $S \lambda>\mathrm{O}\left(E_{V^{\frac{1}{2}}}\right)$, the right-hand side of (3.14) may be neglected and the boundary layer is an inviscid one on the $\bar{t}$ scale. This is the case (2.5c) we have chosen to examine.

The correction variables in the boundary layer are expanded as follows:

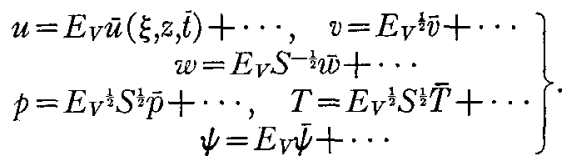

The resulting equations are the same as (3.2) with scaled variables:

$$
\begin{aligned}
-\bar{u}_{\xi}+\bar{w}_{z} & =0, \\
-2 \bar{v}=\bar{p}_{\xi}, & \bar{v}_{\bar{t}}+2 \bar{u}=0, \\
\bar{T}=\bar{p}_{z}, \quad \bar{T}_{\bar{t}}+4 \bar{w} & =0 .
\end{aligned}
$$

The alongshore flow is in geostrophic balance [(3.16b)] and this, along with the hydrostatic equation (3.16d), 
results in the "thermal wind" relation

$$
\bar{v}_{z}=-\frac{1}{2} \bar{T}_{\xi} .
$$

Also, we find, of course, that the potential vorticity is conserved:

$$
\left(-\bar{v}_{\xi}+\frac{1}{2} \bar{T}_{z}\right)_{\bar{t}}=0 \text {. }
$$

It is again convenient to work with the streamfunction $\bar{\psi}$, defined so that $\bar{u}=\bar{\psi}_{z}$ and $\bar{w}=\bar{\psi}_{\xi}$. The equation for $\bar{\psi}$ is

$$
\bar{\psi}_{\xi \xi}+\bar{\psi}_{z z}=0 \text {, }
$$

and the boundary conditions are

$$
\begin{array}{rr}
\bar{\psi}(z=1)=0, \quad \bar{\psi}_{i}(z=0)=\bar{\psi}_{z}(z=0), & (3.20 \mathrm{a}, \mathrm{b}) \\
\bar{\psi}(\xi=0)=-\psi_{0}(x=1), \quad \bar{\psi}(\xi \rightarrow \infty) \rightarrow 0, & (3.20 \mathrm{c}, \mathrm{d})
\end{array}
$$

where $\psi_{0}$ in $(3.20 \mathrm{c})$ is found from (3.13a). A formal solution for the Laplace transform of $\bar{\psi}$ can be obtained, but cannot be readily inverted for general values of $\bar{t}$. It is possible, however, to obtain approximate analytical expressions for $\bar{\psi}$ when $\bar{t} \ll<1$ and when $\bar{t} \gg 1$.

For $\bar{t} \ll 1$, the boundary conditions $(3.20 \mathrm{~b}, \mathrm{c})$ can be approximated by

$$
\bar{\psi}(z=0) \sim 0, \quad \bar{\psi}(\xi=0) \sim \frac{1}{2} \tau_{T}(x=1) z, \quad(3.21 \mathrm{a}, \mathrm{b})
$$

and the solution is

$$
\bar{\psi} \sim \pi^{-1} \tau_{T}(x=1) \tan ^{-1}\left[\frac{\exp (-\pi \xi) \sin \pi z}{1+\exp (-\pi \xi) \cos \pi z}\right] .
$$

The corresponding solutions for $\bar{v}$ and $\bar{T}$ are given by $\bar{v} \sim-2 \bar{\psi} z \bar{t}, \bar{T} \sim-4 \bar{\psi} \bar{\xi} \bar{t}$, or

$$
\begin{gathered}
\bar{v} \sim-2 \tau_{T^{\prime}}(x=1) F^{-1} \exp (-\pi \xi) \\
\times[\cos \pi z+\exp (-\pi \xi)] \bar{t}, \\
\bar{T} \sim 4 \tau_{T}(x=1) F^{-1} \exp (-\pi \xi) \sin (\pi z) \bar{t},
\end{gathered}
$$

where

$$
F=[1+2 \exp (-\pi \xi) \cos \pi z+\exp (-2 \pi \xi)] .
$$

A plot of $-i^{-1}$ times the total alongshore velocity in the boundary layer, $-\bar{t}^{-1}\left[\bar{v}(\xi)+v_{0}(x=1)\right]$, for various values of $z$, is given in Fig. 1 [recall $\tau_{T}(x=1)=-1$ ] . Note that, except at $z=1, \bar{v}(\xi=0)+v_{0}(x=1)=0$, resulting from the condition $u(x=1)=0[(3.2 c)$ and (3.16c) $]$. At $z=1, \bar{v}(z=1, \xi \rightarrow 0)$ is unbounded, corresponding to the singularity in $\bar{\psi}_{z}(z=1, \xi \rightarrow 0)$, which reflects the concentrated inflow into the surface Ekman layer through the corner region. The inclusion of viscous effects would eliminate the singularity in $\bar{v}(z=1, \xi \rightarrow 0)$. The formation of a coastal jet near the top surface is clearly illustrated in Fig. 1. The axis of the jet (line of maximum velocity) is given by the points where $\bar{\psi}_{z \xi}=0$. Near the coast $\left(1-z \ll 1,{ }^{\prime} \xi \ll 1\right)$, the axis lies along the line $\xi \sim(1-z)$.

An explanation of the physics of the jet is as follows. The nonzero surface stress at $x=1$ causes a flow in the surface Ekman layer away from the coast. To balance

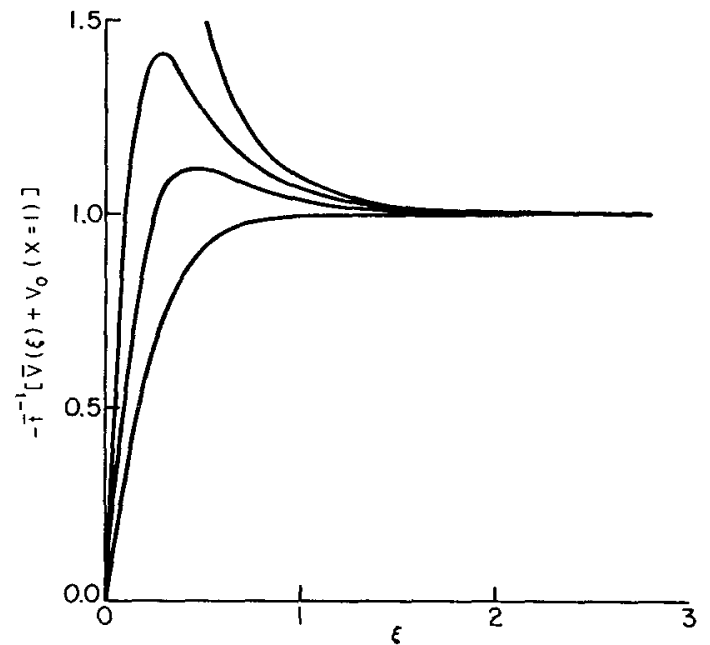

FIG. 1. The total alongshore velocity, for $i \ll 1$, multiplied by $-\bar{t}^{-1},-\dot{t}^{-1}\left[\tilde{v}(\xi)+v_{0}(x=1)\right]$, in the upwelling layer as a function of $\xi$ for $z=0.5,0.65,0.75,1.0$, where increasing values of $z$ correspond to increasing maximum values.

this outflow, fluid upwells into the surface Ekman layer through the corner regions. The upwelling fluid comes through an $\mathrm{O}\left(S^{\frac{1}{2}}\right)$ boundary layer, because in this thin layer the stratification, even though it is weak, can act to break the constraints (3.12) that are present in the interior, and allow the "thermal wind" balance $[(3.17)]$ and the conservation of potential vorticity [(3.18)]. The inflow to the upwelling layer, from the interior, is distributed, for $\bar{t} \ll 1$, uniformly with depth, as can be seen from $(3.21 \mathrm{~b})$. The vertical upwelling velocities $\bar{w}$ in the boundary layer change $\bar{T}[(3.16 \mathrm{e})]$ and the onshore velocities $\bar{u}$ change $\bar{v}[(3.16 \mathrm{c})]$. At the same time $\bar{w}$ and $\bar{u}$ are coupled by the continuity equation (3.16a) and by the "thermal wind" equation (3.17) which couples the changes in $\bar{v}$ and $\bar{T}$ and which, with (3.16c,e), yields (3.19). This results in a baroclinic alongshore flow $\bar{v}$ which reflects the structure of the onshore flow $\bar{u}$. Since the total onshore flow $u_{0}+\bar{u}$ in the upwelling layer evidently speeds up near the top surface as the fluid converges into the upwelling corner regions, the total alongshore flow $v_{0}+\bar{v}$ has a region of relatively high velocity near the coast.

The above analysis for $\bar{t} \ll 1$ only indicates the initial, small-time development of the coastal jet. It corresponds to the analytical description, for small times, of the coastal jet in two-layer fluid models (Charney, 1955; Csanady, 1968).

For $\bar{t} \gg 1$, the interior flow (3.13) is given by the quasi-steady state

$$
\begin{aligned}
& \psi_{0} \sim-\frac{1}{2} \tau_{T}(x), \\
& v_{0} \sim \tau_{T}(x),
\end{aligned}
$$

which is the steady solution for a completely homogeneous fluid. There is an Ekman layer on the bottom surface which receives, by a $z$-independent interior vertical velocity, any interior outflow from the surface Ekman 
layer. The flow from the interior to the upwelling layer is now entirely through the bottom Ekman layer and not through the interior. The solution for $\bar{\psi}$ in the boundary layer can be found from (3.19) with (3.20a,d) and with boundary conditions $(3.20 \mathrm{~b}, \mathrm{c})$ replaced by

It is

$$
\bar{\psi}_{z}(z=0) \sim 0, \quad \bar{\psi}(\xi=0) \sim \frac{1}{2} \tau_{T^{\prime}}(x=1) . \quad(3.24 \mathrm{a}, \mathrm{b})
$$

$$
\bar{\psi} \sim \pi^{-1} \tau_{T}(x=1) \tan ^{-1}\left[\frac{\sin \frac{1}{2} \pi(z+1)}{\sinh \frac{1}{2} \pi \xi}\right] .
$$

This represents an upwelling circulation from the bottom Ekman layer upward and into the corner regions and the surface Ekman layer at the coast.

The alongshore velocity and the temperature may be written as

where

$$
\bar{v} \sim \bar{v}_{T}+\bar{v}_{H}(\xi, z), \quad \bar{T} \sim \bar{T}_{T}+\bar{T}_{I I}(\xi, z)
$$

$$
\bar{v}_{T}=-2 \bar{\psi}_{z} \bar{t}, \quad \bar{T}_{T}=-4 \bar{\psi}_{\xi} \bar{t} .
$$

The first components $\bar{v}_{T}$ and $\bar{T}_{T}$, which are growing linearly with $\bar{t}$, may be found from (3.25) and will be discussed shortly. The second components $\bar{v}_{H}$ and $\bar{T}_{H}$ have reached a quasi-steady state; $\bar{v}_{H}$ adjusts the interior velocity (3.23b) so that the total alongshore velocity is equal to zero at the coast and it also provides the horizontal derivative to satisfy the Ekman layer suction condition (2.6b) at $z=0$.

It appears at first that $\bar{v}$ is not uniquely determined (and similarly for $\bar{T}$ ), in that $\bar{v}_{T}$ could be written $\bar{v}_{T}=-2 \bar{\psi}_{z}\left(\bar{t}+C_{0}\right)$, where $C_{0}$ is an arbitrary constant. However, a check of the full solution (3.10), which is valid for all $\vec{t}$, indicates that $C_{0}=0$.

It is useful, in obtaining $\bar{v}_{H}$ and $\bar{T}_{H}$, to note that the time integration of (3.18) gives

$$
\bar{v}_{\xi}=\frac{1}{2} \bar{T}_{z} \text {. }
$$

It follows from (3.28) and (3.17) that $\left(\bar{v}, \frac{1}{2} \bar{T}\right)$ are conjugate harmonic functions. Consequently, $\bar{v}$ and therefore $\bar{v}_{H}$ satisfy

$$
\bar{v}_{H \xi \xi}+\bar{v}_{H z z}=0 .
$$

The boundary conditions for $\bar{v}_{H}$ are

$$
\begin{gathered}
\bar{v}_{H z}(z=1)=0, \quad \bar{v}_{H}(z=0)=-2 \bar{\psi}(z=0), \\
\bar{v}_{H}(\xi=0)=-\tau_{T^{\prime}}(x=1), \quad \bar{v}_{H}(\xi \rightarrow \infty) \rightarrow 0 .
\end{gathered}
$$

The solution is

where

$$
\bar{v}_{H}=-2(1-z) \bar{\psi}+\bar{v}_{H 1}+\bar{v}_{I I 2},
$$

$\left.\begin{array}{l}\bar{v}_{H 1}=-2 \tau_{T}(x=1) \sum_{n=1}^{\infty}(-1)^{n+1} k_{n}{ }^{-2} \exp \left(-k_{n} \xi\right) \sin k_{n} z \\ \bar{v}_{H 2}=-2 \tau_{T}(x=1) \xi \sum_{n=1}^{\infty}(-1)^{n+1} k_{n}{ }^{-1} \exp \left(-k_{n} \xi\right) \sin k_{n} z\end{array}\right\}$,

and where $k_{n}=\frac{1}{2} \pi(2 n-1) . \bar{T}_{H}$ can be obtained from $\bar{v}_{H}$ by utilizing (3.17) and (3.28).

The components $\bar{v}_{T}$ and $\bar{T}_{T}$, which are growing linearly with $\bar{t}$, are directly forced by the quasi-steady values, for $\bar{l} \gg 1$, of $\bar{u}$ and $\bar{w}$. For large values of $\bar{t}, \bar{v}_{r}$ and $\bar{T}_{T}$ will, in general, be larger in magnitude than the quasi-steady components $\bar{v}_{I I}$ and $\bar{T}_{H}$ and are therefore of more interest to us. From (3.25) and (3.27) we obtain

$$
\begin{gathered}
\vec{v}_{T}=-\tau_{T}(x=1) G^{-1} \cos \left[\frac{1}{2} \pi(z+1)\right] \sinh \left(\frac{1}{2} \pi \xi\right) \bar{t}, \\
\bar{T}_{T}=2 \tau_{T}(x=1) G^{-1} \sin \left[\frac{1}{2} \pi(z+1)\right] \cosh \left(\frac{1}{2} \pi \xi\right) \bar{t},
\end{gathered}
$$

where

$$
G=\left\{\sinh ^{2}\left(\frac{1}{2} \pi \xi\right)+\sin ^{2}\left[\frac{1}{2} \pi(z+1)\right]\right\} .
$$

Plots of $-\bar{t}^{-1} \bar{v}_{\eta}$, for various values of $z$, are given in Fig. 2 and show that $\bar{v}_{r}$ exhibits the structure of a coastal jet. The axis of the jet, near the coast $(1-z \ll 1$, $\xi \ll 1)$, is along the line $\xi \sim(1-z)$. The features of the jet, which are most pronounced near the surface, extend now to a greater depth than was the case for $\dot{k} \ll 1$.

We see, therefore, that, for $\bar{l} \gg 1$, there is a coastal jet in the $\mathrm{O}\left(S^{\frac{1}{2}}\right)$ upwelling layer with an alongshore velocity component that is growing linearly with $\bar{t}$. This result is basically different than those from linear two-layer models. In that case, the fluid, if $A_{V}$ is assumed to be a constant throughout, should spin up by the same basic mechanisms described by Pedlosky (1967). The two-layer flow would, therefore, reach a steady state as $t \rightarrow \infty$, i.e., on the $t=\mathrm{O}\left(E_{V}{ }^{-\frac{1}{2}}\right)$ time scale. A solution for the steady-state interior flow, outside of side-wall $E_{H^{\frac{1}{4}}}$ frictional layers, may be found easily [e.g., by a slight modification of the procedure of Carrier (1965)] and does not exhibit a coastal jet structure. Evidently, therefore, in linear two-layer models, with a constant value of $A_{V}$, the coastal jet disappears on the $t=\mathrm{O}\left(E_{V}{ }^{-\frac{1}{2}}\right)$ time scale. This difference in behavior, regarding time scales, has implications, of course, for oceanic upwelling regions and these are discussed in Section 5.

\section{The diffusive adjustment of the coastal jet: $t=\mathrm{O}\left(S E_{H}{ }^{-1}\right)$}

Now that upwelling and the development of a coastal jet have been described on the $t=O\left(E_{V}^{-\frac{1}{2}}\right)$ time scale, it is of interest to inquire into the fate of the jet as time increases and into the nature of the approach of the flow to a steady state.

From (3.14), we can see that horizontal diffusion will be important in the $\mathrm{O}\left(S^{\frac{1}{2}}\right)$ upwelling layer on a time scale of $O\left(S E_{I I}^{-1}\right)$. To investigate the flow on this time scale, therefore, we define a new time variable

$$
t=\left(S E_{H}^{-1}\right) \tilde{t} .
$$

The asymptotic form, for $t \gg 1$, of the boundary layer variables (3.26) indicates the magnitude of $v$ and $T$ for the $\tilde{t}$ scale, i.e.,

$$
\begin{aligned}
& v=E_{V^{\frac{1}{2}} \bar{v}} \sim E_{V^{\frac{1}{2}} \bar{t}}=S \lambda \tilde{t},
\end{aligned}
$$

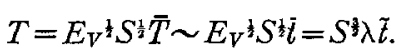


Guided by (4.2), we expand the correction variables in the $O\left(S^{\frac{1}{2}}\right)$ layer as follows:

$\left.\begin{array}{l}u=E_{V} \tilde{u}(\xi, z, \tilde{l})+\cdots, \quad v=S \lambda \tilde{v}+\cdots, \quad v=E_{V} S^{-\frac{1}{2}} \tilde{v} \\ p=S^{\frac{3}{2}} \lambda \tilde{p}+\cdots, \quad T=S^{\frac{3}{2}} \lambda \tilde{T}+\cdots, \quad \psi=E_{V} \tilde{\psi}+\cdots\end{array}\right\}$.

The resulting equations are

$$
\begin{gathered}
-\tilde{u}_{\xi}+\tilde{v}_{z}=0, \\
-2 \tilde{v}=\tilde{p}_{\xi}, \quad \tilde{v}_{t}+2 \tilde{u}=\tilde{v}_{\xi \xi}+S \lambda \tilde{v}_{z z}, \\
\tilde{T}=\tilde{p}_{z}, \quad \tilde{T}_{i}+4 \tilde{v}=\sigma^{-1}\left(\tilde{T}_{\xi \xi}+S \lambda \tilde{T}_{z z}\right),
\end{gathered}
$$

where, in particular, it again follows that

$$
\tilde{v}_{z}=-\frac{1}{2} \widetilde{T}_{\xi} .
$$

For $S \lambda<\mathrm{O}(1)$, the viscous and heat conduction terms, involving the $z$ derivatives, are small and may be neglected. If $S \lambda=\mathrm{O}(1)$, however, they are important and must be retained. We include these terms and the resulting solutions will be valid for $S \lambda \leqslant O(1)[(2.5 c)]$.

One equation may be derived for $\tilde{p}$, i.e.,

$$
\left(\tilde{p}_{\xi \xi}+\widetilde{p}_{z z}\right)_{l}=\tilde{p}_{\xi \xi \xi \xi}+\left(S \lambda+\sigma^{-1}\right) \tilde{p}_{\xi \xi z z}+S \lambda \sigma^{-1} \tilde{p}_{z z z z} \text {. }
$$

Two boundary conditions are required for $\tilde{p}$ at the top and at the bottom surface. At $z=0$, it follows from (2.6b) that $\tilde{v}(z=0)=0$ and from the temperature boundary condition $(2.4 \mathrm{c})$ that $\widetilde{T}_{z}(z=0)=0$. In terms of the pressure, these conditions are $\widetilde{p}(z=0)=\widetilde{p}_{z z}(z=0)$ $=0$. Note also that these boundary conditions imply $\tilde{u}(z=0)=0$ from $(4.4 \mathrm{c})$.

At $z=1$, one condition on $\widetilde{p}$ results directly from the temperature boundary condition $(2.4 \mathrm{~d}), \quad \tilde{T}(z=1)$ $=\tilde{p}_{z}(z=1)=0$, where, from (4.5), this implies $\tilde{v}_{z}(z=1)$ $=0$. Let us consider for a moment, however, a more general situation $\tilde{T}(z=1) \neq 0$. In that case, $\tilde{v}_{z}(z=1) \neq 0$ and an Ekman layer, with an alongshore velocity component $v_{E}$, must be present to satisfy the stress boundary condition, i.e.,

$$
S \lambda \tilde{y}_{z}(z=1)+v_{E z}(z=1)=0,
$$

or, with (4.5),

$$
v_{E z}(z=1)=\frac{1}{2} S \lambda \widetilde{T}_{\xi}(z=1) .
$$

This results in an Ekman layer suction velocity of $\mathrm{O}\left(S \lambda E_{V} S^{-\frac{1}{2}}\right)$ and, therefore, in a condition on $\tilde{\mathcal{w}}$ :

$$
\tilde{\mathcal{w}}(z=1)=-\frac{1}{2} S \lambda \tilde{T}_{\xi \xi}(z=1) .
$$

A condition on $\tilde{p}$ may be derived from (4.7) using $(4.4 \mathrm{~d}, \mathrm{e})$. In our case, $(4.7)$ yields $\widetilde{w}(z=1)=0$. It then follows from $(4.4 \mathrm{~d}, \mathrm{e})$ that $\widetilde{T}_{z z}(z=1)=\tilde{p}_{z z z}(z=1)=0$.

The initial condition for the streamfunction is given by (3.25), i.e., $\tilde{\psi}(\tilde{t} \rightarrow 0)=\bar{\psi}(\bar{t} \rightarrow \infty)$. As $\tilde{t} \rightarrow \infty$, the streamfunction $\tilde{\psi}$ will approach a steady state, $\tilde{\psi} \rightarrow \tilde{\psi}_{S}(\xi, z)$. In the steady state, the governing equation for $\tilde{\psi}_{s}$, from (4.4), is

$$
\tilde{\psi}_{s \xi \xi}+\sigma^{-1} \tilde{\psi}_{s z z}=0 .
$$

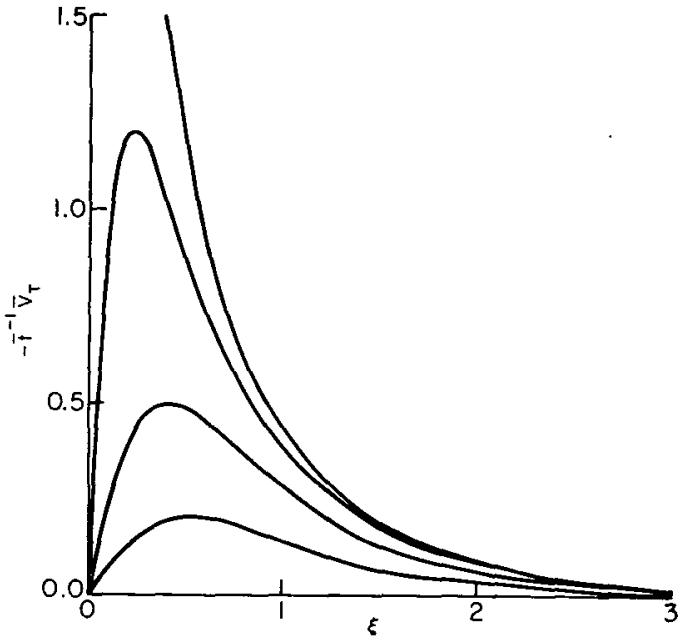

FIG. 2. The alongshore velocity component $\bar{v}_{T}$, for $i \gg 1$, multiplied by $-\bar{t}^{-1},-\bar{t}^{-1} \bar{v}_{T}$, as a function of $\xi$ for $z=0.25,0.5,0.75,1.0$, where increasing values of $z$ correspond to increasing maximum values.

[Note, one equation for the streamfunction alone does not follow for $S \lambda=\mathrm{O}(1)$, if $\left.\sigma_{H} \neq \sigma_{V}.\right]$ The boundary: conditions for $\tilde{\psi}_{S}$ are

$$
\begin{gathered}
\tilde{\psi}_{S}(z=1)=0, \quad \tilde{\psi}_{S z}(z=0)=0, \\
\tilde{\psi}_{S}(\xi=0)=\frac{1}{2} \tau_{T}(x=1), \quad \tilde{\psi}_{S}(\xi \rightarrow \infty) \rightarrow 0 .
\end{gathered}
$$

Conditions (4.9) are identical to (3.20a,d) and (3.24a,b). Because of this and the similarity of (3.19) and (4.8), it is clear that on the $\tilde{t}$ time scale $\tilde{\psi}$ will adjust through diffusive processes to a new structure which is qualitatively identical to its original structure, but which has a different boundary layer thickness of $\mathrm{O}\left(\sigma^{\frac{1}{2}} S^{\frac{1}{2}}\right)$. This steady-state boundary layer is the hydrostatic layer (Barcilon and Pedlosky, 1967). Since for $\sigma=O(1)$ there are no major changes in the structure of $\tilde{\psi}$ on the $\tilde{t}$ scale, it appears that the important features of the adjustment of $\tilde{v}$ and $\tilde{T}$ will still be retained if we consider the special case

$$
\sigma=1 .
$$

We assume (4.10) at this point, since it considerably simplifies the description of the problem for $\tilde{v}$ and $\tilde{T}$ and evidently retains the essential physics. The main difference is that with $\sigma=1$ the streamfunction reaches a final steady state on the $\bar{t}$ scale, $\tilde{\psi} \equiv \tilde{\psi}_{S}=\bar{\psi}(\bar{t} \rightarrow \infty)$, whereas otherwise it would adjust simultaneously with $\tilde{v}$ and $\tilde{T}$ on the $\tilde{t}$ scale. With (4.10), therefore, $\tilde{u}$ and $\tilde{w}$ are independent of $\tilde{t}$ and may be obtained from (3.25). Consequently, $\tilde{v}$ and $\tilde{T}$ may be solved for directly from $(4.4 \mathrm{c})$ and $(4.4 \mathrm{e})$ and it is not necessary to consider (4.6).

In the interior, it follows from (3.2e) that $T=\mathrm{O}\left(S E_{V}\right) t$, which, through the "thermal wind" relation, implies that, in addition to $(3.23 \mathrm{~b})$, $v$ has a component $v=\mathrm{O}\left(S E_{V}\right) t$. For $t=\mathrm{O}\left(S E_{H}{ }^{-1}\right)$ we obtain $T, v=\mathrm{O}\left(S^{2} \lambda\right)$. The magnitudes in the interior of $v$ and 
$T_{x}$ are, therefore, smaller than the magnitudes of the boundary layer variables, and $\tilde{v}$ and $\widetilde{T}$ must satisfy the boundary conditions

$$
\tilde{v}(\xi=0)=0, \quad \tilde{T}_{\xi}(\xi=0)=0 .
$$

The other boundary conditions for $\tilde{v}$ are

$$
\tilde{v}_{z}(z=1)=0, \quad \tilde{v}(z=0)=0, \quad \tilde{v}(\xi \rightarrow \infty) \rightarrow 0,
$$

and those for $\tilde{T}$

$\tilde{T}(z=1)=0, \quad \widetilde{T}_{z}(z=0)=0, \quad \tilde{T}(\xi \rightarrow \infty) \rightarrow 0 . \quad(4.13 \mathrm{a}, \mathrm{b}, \mathrm{c})$

In what follows, we concentrate mainly on a description of the development of $\tilde{v}$. The solution for $\tilde{T}$ can be found along similar lines. The problem for $\tilde{v}$ is given by Eq. (4.5c), where $\tilde{u}=\tilde{\psi}_{z}$ is found from (3.25), with boundary conditions (4.11a) and (4.12). For the initial condition, $\tilde{v}(\tilde{t} \rightarrow 0)$ must match with the $\mathrm{O}(\mathrm{S} \lambda)$ component of $\bar{v}(\vec{t} \rightarrow \infty)$, i.e.,

$$
S \lambda \tilde{v}(\tilde{t} \rightarrow 0)=E_{V^{\frac{3}{2}}} \bar{v}_{1^{\prime}}(\dot{t} \rightarrow \infty) \sim S \lambda \tilde{t},
$$

which results in

$$
\tilde{v}(\tilde{t}=0)=0 .
$$

It is clear that the solution to (4.4c) for $\tilde{v}$, with (4.11a), (4.12) and (4.14), will contain $\bar{v}_{T}$ [and other higher order corrections to $\bar{v}$ on the $\bar{t}$ scale which become $\mathrm{O}(S \lambda)$ for $\bar{t} \rightarrow \infty]$ and that $\bar{v}_{T}$ will be an approximate representation of $\tilde{v}$ for $\tilde{t} \ll 1$.

The problem for $\tilde{v}$ has the same form as a twodimensional problem in heat conduction with an interior heat source represented by $\tilde{u}$; intuition concerning the behavior of solutions to heat conduction problems may be used here to visualize the results. The solution may be obtained with the aid of the Laplace transform and is

where

$$
\tilde{v}=\tilde{v}_{S}+\tilde{v}_{1}+\tilde{v}_{2}
$$

$$
\begin{aligned}
\tilde{v}_{S}= & -2 \tau_{T}(x=1)(1-S \lambda)^{-1} \sum_{n=1}^{\infty}(-1)^{n+1} k_{n}{ }^{-2} \\
& \times \exp \left(-k_{n} \xi\right) \sin k_{n} z, \\
\tilde{v}_{1}= & 2 \tau_{T}(x=1)(1-S \lambda)^{-1} \sum_{n=1}^{\infty}(-1)^{n+1} k_{n}{ }^{-2} \\
& \times \exp \left[k_{n}^{2}(1-S \lambda) \tilde{t}\right] \\
& \times\left\{\exp \left(-k_{n} \xi\right)-\frac{1}{2}\left[\exp \left(k_{n} \xi\right) \operatorname{erfc}\left(\frac{1}{2} \xi \tilde{t}^{-\frac{1}{2}}+k_{n} \tilde{t}^{\frac{1}{2}}\right)\right.\right. \\
& \left.\left.\quad+\exp \left(-k_{n} \xi\right) \operatorname{erfc}\left(\frac{1}{2} \xi \tilde{t}^{-\frac{1}{2}}-k_{n} \tilde{t}^{\frac{1}{2}}\right)\right]\right\} \sin k_{n} z \\
\tilde{v}_{2}= & \tau_{T}(x=1)(1-S \lambda)^{-1} \\
& \times \sum_{n=1}^{\infty}(-1)^{n+1} k_{n}{ }^{-2}\left\{\exp \left[(S \lambda)^{\frac{1}{2}} k_{n} \xi\right]\right. \\
& \quad \times \operatorname{erfc}\left[\frac{1}{2} \xi \tilde{t}^{-\frac{1}{2}}+k_{n}(\lambda S)^{\frac{1}{2} \tilde{t}^{\frac{1}{2}}}\right]+\exp \left[-(S \lambda)^{\left.\frac{1}{2} k_{n} \xi\right]}\right. \\
& \quad \times \operatorname{erfc}\left[\frac{1}{2} \xi \tilde{t}^{-\frac{1}{2}}-k_{n}(\lambda S)^{\left.\frac{1}{2} \tilde{t}^{\frac{1}{2}}\right]}\right] \sin k_{n} z
\end{aligned}
$$

and where

$$
k_{n}=\frac{1}{2} \pi(2 n-1) \quad \text { and } \quad \operatorname{erfc}(x)=2 \pi^{-\frac{2}{2}} \int_{x}^{\infty} \exp \left(-y^{2}\right) d y .
$$

The three parts of the solution may be interpreted in the following way: $\tilde{v}_{s}$ is the final steady-state solution in the hydrostatic layer. The governing equations for this layer are given by (4.4) with $\partial / \partial \tilde{t} \equiv 0$. The individual variables satisfy $(4.8)$. The steady-state solution for the streamfunction $\tilde{\psi}_{S}$ in the hydrostatic layer is given by (3.25). [For general values of $\sigma$, substitute $\xi \rightarrow \sigma^{-\frac{1}{2}} \xi$ and $(1-S \lambda) \rightarrow \sigma^{-1}(1-\sigma \lambda S)$ in (3.25) and (4.15b).] The velocity $\tilde{v}_{S}$ satisfies the condition.

$$
\tilde{v}_{S}(\xi=0)=\tau_{T}(x=1) \sigma(1-\sigma S \lambda)^{-1} z .
$$

The origin of this may be seen from an integration of the steady-state form of (4.4e) after the substitution of $\tilde{T}_{S z z}=-\sigma \widetilde{T}_{S \xi \xi}$ :

$$
\begin{aligned}
-\int_{0}^{\infty} 4 \tilde{w}_{S} d \xi=4 \tilde{\psi}(\xi=0) & =2 \tau_{T}(x=1) \\
& =\left(\sigma^{-1}-S \lambda\right) \widetilde{T}_{S \xi}(\xi=0) .
\end{aligned}
$$

Eq. (4.16a) results from the use of (4.16b) and (4.5), an integration with respect to $z$, and an application of $(4.12 \mathrm{~b})$.

The component $\tilde{v}_{1}$ is a solution to the problem

$$
\begin{gathered}
\tilde{v}_{1 t}=\tilde{v}_{1 \xi \xi}+(S \lambda) \tilde{v}_{1 z z}, \\
\tilde{v}_{1}(\tilde{t}=0)=-\tilde{v}_{S}, \quad \tilde{v}_{1}(\xi=0)=0 \quad(\tilde{l}>0), \\
\tilde{v}_{1}(\xi \rightarrow \infty) \rightarrow 0 .
\end{gathered}
$$

and $(4.12 a, b)$. This just represents the diffusive decay of an initial distribution (4.18a) subject to the homogeneous boundary conditions $(4.18 \mathrm{~b}, \mathrm{c})$.

The component $\tilde{v}_{2}$ satisfies the problem given by Eq. (4.17) with boundary conditions

$$
\begin{gathered}
\tilde{v}_{2}(\tilde{t}=0)=0, \quad \tilde{v}_{2}(\xi=0)=-\tilde{v}_{S}(\xi=0)(\tilde{t}>0), \\
\tilde{v}_{2}(\xi \rightarrow \infty) \rightarrow 0 .
\end{gathered}
$$

and $(4.12 \mathrm{a}, \mathrm{b})$. This corresponds to the diffusive adjustment, in a two-dimensional region with zero initial values, to an impulsively applied nonzero end value (4.19b) at $\xi=0$. From (4.15d), we can see that this solution reaches a steady state, given by

$$
\begin{array}{r}
\tilde{v}_{2 S}=2 \tau_{T}(x=1)(1-S \lambda)^{-1} \sum_{n=1}^{\infty}(-1)^{n+1} k_{n}{ }^{-2} \\
\times \exp \left[-(S \lambda)^{\left.\frac{1}{2} k_{n} \xi\right] \sin k_{n} z},\right.
\end{array}
$$

on a time scale $\tilde{t}=\mathrm{O}(\lambda S)^{-1}$ or $t=\mathrm{O}\left(E_{V}{ }^{-1}\right)$. [For general values of $\sigma$, substitute $(1-S \lambda) \rightarrow \sigma^{-1}(1-\sigma S \lambda)$ only in (4.20).] The steady solution is confined, for $\lambda \gg 1$, to a boundary layer of width $\delta_{x 2}=\mathrm{O}\left(\lambda^{-\frac{1}{2}}\right)$, which we will refer to as the diffusion layer. Note that $\tilde{v}_{2 S}$ and $\tilde{T}_{2 S}$ 
satisfy the equation

$$
\tilde{v}_{2 S \xi \xi}+S \lambda \tilde{\nu}_{2 S z z}=0
$$

and there are, in this particular problem, no upwelling velocities $\tilde{u}$ and $\tilde{w}$ involved in the diffusion layer. The magnitude of $\tilde{T}_{2 S}$ is $\mathrm{O}\left(\lambda^{-\frac{1}{2}} S^{-1}\right)$, i.e., $T=\mathrm{O}\left(S \lambda^{\frac{1}{2}}\right)$.

The steady-state form of (4.6) may be written as

where

$$
\nabla_{1}^{2} \nabla_{2}^{2} \tilde{p}=0
$$

$$
\begin{aligned}
& \nabla_{1}{ }^{2}=\left(\partial^{2} / \partial \xi^{2}+\sigma^{-1} \partial^{2} / \partial z^{2}\right), \\
& \nabla_{2}{ }^{2}=\left(\partial^{2} / \partial \xi^{2}+S \lambda \partial^{2} / \partial z^{2}\right) .
\end{aligned}
$$

Evidently, with our particular choice of boundary conditions for $\widetilde{T}$ and $\tilde{v}$, which allow an expansion of $\widetilde{p}$ in $\sin k_{n} z, \tilde{p}$ is the sum of two solutions $\tilde{p}_{S}$ and $\tilde{p}_{2 S}$ which satisfy $\nabla_{1}^{2} \widetilde{p}_{S}=0$ and $\nabla_{2}^{2} \tilde{p}_{2 S}=0$, respectively.

Solutions for $v=S \lambda \tilde{v}$, at various values of $z$ and $\tilde{t}$, and for different values of $S \lambda$, are plotted in Fig. 3. The initial formation of the jet for $\tilde{i} \ll 1$, which is similar to that given by (3.31a), is illustrated. Also shown is the diffusive widening of the jet, as $\tilde{t}$ increases, and the eventual approach to a steady state in the $S^{\frac{1}{2}}$ and $\lambda^{-\frac{1}{2}}$ boundary layers. The slower approach to a steady state, for smaller values of $S \lambda$, can be seen.

It is of interest to see how the upwelling circulation drives the adjustment of $\tilde{v}$. The process is inviscid initially, for short times, and then subsequently is governed by diffusion. Note that the inviscid flow satisfies the boundary conditions for $\tilde{v}$ and that therefore diffusion does not enter to adjust boundary values, but rather to limit and modify the growth of $\tilde{v}$ due to $\tilde{u}$.

The steady-state values of $v$ at various values of $z$, for $S \lambda=0.5$, are plotted in Fig. 4 . A jet-like structure is evident. Near the top surface there is relatively little variation with $z$, which reflects the boundary condition $v_{z}(z=1)=0[(4.12 \mathrm{a})]$. In Fig. 5, $v(z=1)$ is compared for different values of $S \lambda$. We can see that for $S \lambda=0.9$ the hydrostatic and diffusion layers are merged and there exists a steady state coastal current which is confined to a narrow region. For $S \lambda=0.1$, however, the layers are separated, to some extent, and the coastal current, which is smaller in magnitude, is diffused over a larger region. We also point out that if $S \lambda \ll 1$, the alongshore velocity at the edge of the hydrostatic layer has a linear variation with $z$ given by (4.16a).

In the interior, the temperature adjusts in general on the $t=\mathrm{O}\left(E_{V}{ }^{-1}\right)$ time scale. For $\lambda \gg 1$, the steady state value is given approximately by the solution to

$$
-4 S \psi_{0 x}=2 S_{\tau_{T x}}=\sigma^{-1} T_{I S z z},
$$

with boundary conditions [from $(2.4 c, d)]$

$$
T_{I S}=\sigma S_{\tau_{T x}}\left(z^{2}-1\right) \text {. }
$$

The interior alongshore velocity, which has an $\mathrm{O}\left(E_{V^{\frac{1}{2}}}\right)$ component given by (3.23b), will also have a baroclinic component which may be obtained with (4.24) and an
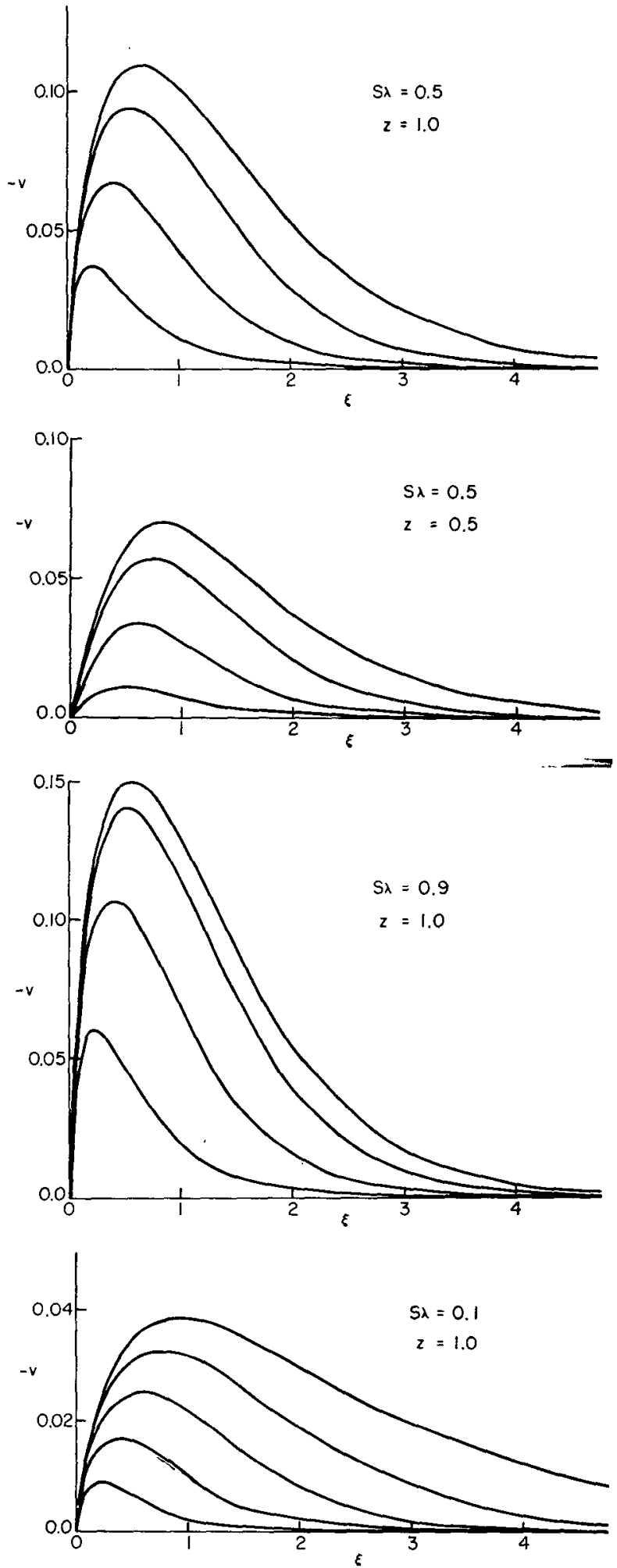

FIG. 3. The alongshore velocity $v=S \lambda \tilde{v}$ as a function of $\xi$ for different values of $\vec{t}$ and for the values of $S \lambda$ and $z$ marked on the plots. The values of $\tilde{t}$ are $\tilde{t}=0.05,0.2,0.6, \infty$ (steady state), with the exception of $S \lambda=0.1$ where $\bar{t}=1.2$ is also included. Increasing values of $\tilde{t}$ correspond to increasing maximum values. 


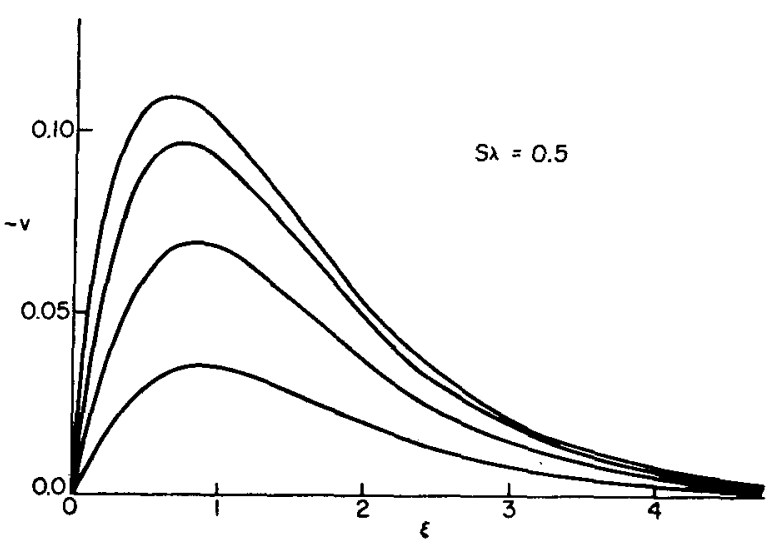

Fig. 4. The steady-state values of $\nu=S \lambda \tilde{v}$ as a function of $\xi$ for $S \lambda=0.5$ and for $z=0.25,0.5,0.75,1.0$, where increasing values of $z$ correspond to increasing maximum values.

integration of the thermal wind equation. This component will have a magnitude $O(S)$. The important point here is that, with conditions (2.5), the values of the alongshore velocity in the interior are much smaller than those in the coastal layers. Therefore, there is a coastal current, or jet, with relatively large alongshore velocities in both the transient flow and in the final steady state. The width of the coastal current in the initial time-dependent phase is $O\left(S^{\frac{1}{2}}\right)$ and the width in the steady state is $O\left(\lambda^{-\frac{1}{2}}\right)$.

\section{Discussion}

A simple, linear, $f$-plane model has been used here in an attempt to describe upwelling and the formation of a coastal jet in a continuously stratified ocean. We should emphasize that, even with this idealized geometry and within the framework of linear theory, the problem here has been further specialized by the particular choice of boundary conditions for the temperature $[(4.13 \mathrm{a}, \mathrm{b})]$ and the assumption (2.5b) of equal vertical and horizontal Prandtl numbers. These assumptions were made, of course, to aid in the obtainment of a solution. In particular, these conditions result, for $S \lambda \leqslant O(1)$, in a splitting of the solutions of (4.22) into two components which correspond to solutions for the hydrostatic and diffusion layers. This splitting of solutions to (4.22), with exponential variation in the horizontal direction, is similar to the splitting of solutions to essentially the same equation (Allen, 1972) into interior (diffusion) and Lineykin layer components, with exponential variation in the vertical direction.

In the initial transient phases of the solution [Section 3], the effect of the stratification is clearly measured by the parameter $S$. Investigations of steadystate upwelling in continuously stratified models (Blumsack, 1972; Hsueh and Kenney, 1972; Allen, 1972) have shown, however, that when the flow is steady, the parameter $\sigma S \lambda$ is a measure of the effect of the stratification. The present study shows how, in this particular problem, the parameter $\sigma S \lambda$ emerges during the transient solution and how it becomes important in the steady state.

If a more realistic boundary condition for the temperature at the sea surface were used, it would undoubtably lead, in the upwelling region, to $T(z=1) \neq 0$ and probably to $T_{x}(z=1)<0$ and $T_{x x}(z=1)<0$. From (4.7), we can see that this would result in an upwelling of fluid into the surface Ekman layer in an area which covers the upwelling layer and which is not confined to the corner regions. Note that offshore, past the edge of the upwelling region, the transport in the surface Ekman layer would be unaffected and would have the same value as before, $\frac{1}{2} E_{V} \tau_{T}(x=1)$, assuming no significant variation of the wind stress over the width of the upwelling region. Therefore, the same total amount of water would have to upwell, but it would enter the surface Ekman layer in a different manner.

One feature of the present $f$-plane model that should be pointed out is that, regardless of the temperature boundary conditions or the relative values of the Prandtl numbers, after the adjustment of the flow on the $t=O\left(E_{V}{ }^{-\frac{1}{2}}\right)$ time scale the fluid will be fed from the interior to the coastal region through an Ekman layer on the bottom surface. This somehow conflicts with an intuitive picture of the behavior of oceanic upwelling. The inclusion of the $\beta$-effect changes this feature in homogeneous fluid models (Pedlosky, 1968; Durance and Johnson, 1970; Garvine, 1971) and the fluid is fed from the interior uniformly over the depth. It is not clear at the present to what extent the proper description of oceanic upwelling depends on the $\beta$-effect (Hurlburt and Thompson, 1973) or, if the $\beta$-effect is of fundamental importance, to what extent local $f$-plane solutions might be valid components of a $\beta$-plane model. Let us proceed, however, and assess the scale implications from the present model for the oceanic case.

We remark that the use of constant eddy coefficients to represent the turbulent exchange processes is, of

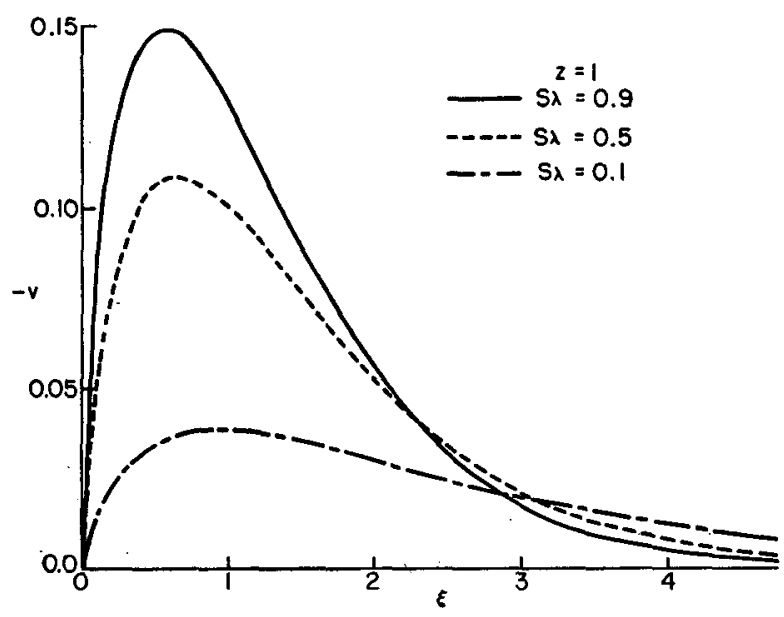

Fic. 5. The steady-state values of $v=S \lambda v$ at $z=1$ as a function of $\xi$ for $S \lambda=0.1,0.5,0.9$. 
course, an assumption of questionable validity. Even if some of the physics is represented thereby, estimates for the appropriate values of the eddy coefficients are very uncertain. It is felt, however, that it is still of value to have an understanding of how a fluid with a NavierStokes-like stress, rate-of-strain relation would behave in the given flow situation.

The primary length scale that emerges here is that of the width of the upwelling region and the coastal jet, $\delta_{x 1}=\mathrm{O}\left(S^{\frac{1}{2}}\right)$. In dimensional variables, this width is

$$
L_{x \mathrm{1}}=\mathrm{O}(H N / 2 \Omega)
$$

the "Rossby radius of deformation," where $H$ is the dimensional depth. The value of $L_{x 1}$ is easy to estimate for upwelling regions, since values of $H, N$ and $2 \Omega$ are known. It has the attractiveness that it is one of the few scales which emerge which do not involve the values of eddy coefficients. If we consider the upwelling region on the continental shelf off the coast of Oregon (Mooers et al., 1973; hereafter referred to as MCS), where the major IDOE field experiment CUE-1 has taken place, we can estimate roughly that $H=200 \mathrm{~m}, N=10^{-2} \mathrm{sec}^{-1}$ (corresponding to a change in density $\Delta \rho / \rho \approx 2 \times 10^{-3}$, over the depth $H$ ), and $2 \Omega=10^{-4} \mathrm{sec}^{-1}$. We find, therefore, that $L_{x 1} \approx 20 \mathrm{~km}$. This length corresponds reasonably well with estimates from observations (MCS) on the width of the near-surface alongshore current or jet.

The requirement that $S \lambda>\mathrm{O}\left(E_{V^{\frac{1}{2}}}\right)$, for the existence of an inviscid upwelling layer of width $O\left(S^{\frac{1}{2}}\right)$ during the $t=\mathrm{O}\left(E_{V}{ }^{-\frac{1}{2}}\right)$ time scale, reduces, in terms of dimensional variables, to

$$
A_{H} A_{V^{-1}}{ }^{-\frac{1}{2}} H N^{2} \Omega^{-\frac{3}{2}} \approx 10^{6} \mathrm{~cm} \mathrm{sec}^{-\frac{1}{2}} .
$$

Here, $A_{V}$ should have a value appropriate for the surface Ekman layer. If we assume $A_{V}=50 \mathrm{~cm}^{2} \mathrm{sec}^{-1}$, we obtain

$$
A_{H} \ll 7 \times 10^{6} \mathrm{~cm}^{2} \mathrm{sec}^{-1} \text {. }
$$

It is possible that this condition is satisfied in upwelling regions. A calculation (MCS) of horizontal eddy coefficients, from measurements of Reynolds stresses and horizontal shear in the Oregon upwelling region, has given values of $A_{H}$ in the range $A_{H} \approx(1-5) \times 10^{5} \mathrm{~cm}^{2}$ $\sec ^{-1}$.

For the purpose of making further estimates we will assume that

$$
\left.\begin{array}{rlrl}
A_{V} & =50 \mathrm{~cm}^{2} \mathrm{sec}^{-1}, & A_{H} & =10^{6} \mathrm{~cm}^{2} \mathrm{sec}^{-1} \\
\tau_{0} & =1 \text { dyn } \mathrm{cm}^{-2}, & \rho_{0} & =1 \mathrm{gm} \mathrm{cm}^{-3} \\
\sigma & =1
\end{array}\right\} .
$$

The other horizontal length scale $\delta_{x 2}=\mathrm{O}\left(\lambda^{-\frac{1}{2}}\right)$, the width of the diffusion layer, has a dimensional value

$$
L_{x 2}=\mathrm{O}\left(H A_{I I^{\frac{2}{2}}} A_{V^{-\frac{1}{2}}}\right) \approx 28 \mathrm{~km} .
$$

This is close to the value for $L_{x 1}$ [corresponding to $\left.S \lambda=A_{V} A_{H}{ }^{-1} N^{2}(2 \Omega)^{-2} \approx 0.5\right]$. The appropriate value of $A_{V}$ for this layer, however, should be that for vertical mixing in the interior, away from the surface Ekman layer. This may well be smaller than our estimate for $A_{V}$ and would result in a larger value for $L_{x 2}$.

An estimate for the horizontal scale of the upwelling corner regions, $\delta_{x 3}=\mathrm{O}\left(E_{H^{\frac{1}{2}}}\right)$, gives

$$
L_{x 3}=\mathrm{O}\left(A_{I I} I^{\frac{1}{3} \Omega^{-\frac{1}{2}}}\right) \approx 1.4 \mathrm{~km} .
$$

With our choice of value for $A_{V}$, the estimated thickness of the Ekman layer, $\delta_{z 1}=\mathrm{O}\left(E_{V^{\frac{1}{2}}}\right)$, is $L_{z 1}$

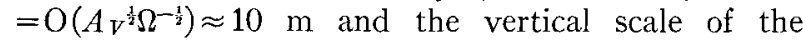
upwelling corner region $[(\mathrm{A} 1 \mathrm{~b})], \delta_{z 2}=\mathrm{O}\left[E_{V^{\frac{1}{2}}}(\sigma S \lambda)^{-\frac{1}{2}}\right]$, is $L_{z 2} \approx 14 \mathrm{~m}$.

Estimates for the time scales $t_{1}=\mathrm{O}\left(E_{V}{ }^{-\frac{1}{2}}\right)$, $t_{2}=\mathrm{O}\left(S E_{H}^{-1}\right)$, and $t_{3}=\mathrm{O}\left(E_{V}^{-1}\right)$ give the following results for the corresponding dimensional times $\left(t_{d}=t \Omega^{-1}\right)$ :

$$
\begin{aligned}
& t_{d 1} \approx H \Omega^{-\frac{1}{2}} A_{V}{ }^{-\frac{1}{2}} \approx 4.6 \text { days } \\
& t_{d 2} \approx H^{2} N^{2}(2 \Omega)^{-2} A_{H H^{-1}} \approx 46.3 \text { days } \\
& t_{d 3} \approx H^{2} A_{V^{-1}} \approx 92.6 \text { days }
\end{aligned}
$$

We see that $t_{d 1}$ is of the order of several days, or some substantial fraction of a week, whereas $t_{d 2}$ is of the order of 6-7 weeks and $t_{d 3}$ is of the order of twice $t_{d 2}$.

Although the values of the time scales $t_{d 1}, t_{d 2}, t_{d 3}$ depend on the uncertain estimates for $A_{V}$ and $A_{H}$, the differences in their magnitudes should be emphasized. In terms of the total length of time $t_{T}$ of the upwelling season off the Oregon coast, say, e.g., three or four months, we have roughly that $t_{d 1} \ll t_{T}$ and $t_{d 2} \approx t_{d 3} \approx t_{T}$. The coastal jet structure forms initially on a time scale of the order of a few days, while it adjusts to a steady state on a time scale which is, evidently, a time of the order of the total upwelling season.

Concerning the predicted magnitude of the currents, the alongshore velocity, on the $t_{1}$ time scale, has a magnitude $v=\mathrm{O}\left(E_{V^{\frac{1}{2}}}\right)$, which gives a dimensional value

$$
v_{d} \approx \tau_{0}\left(\rho_{0} A_{\left.V^{\frac{1}{2}} \Omega^{\frac{1}{2}}\right)^{-1} \approx 20 \mathrm{~cm} \mathrm{sec}^{-1} .}\right.
$$

This compares well with observations (MCS), which have found magnitudes of the alongshore current, near the surface, to be of the order of $20 \mathrm{~cm} \mathrm{sec}^{-1}$.

On the $t_{2}$ time scale, the magnitude of $v$ is larger. To estimate this magnitude, we note from Fig. 5 that the maximum steady value of $v$ in the alongshore current, for $S \lambda=0.5$, is

$$
v=\mathrm{O}\left[\sigma S \lambda(1-\sigma S \lambda)^{-1} \tau_{T}(x=1)\right] \approx 0.1 .
$$

Consequently, the dimensional magnitude is

$$
v_{d} \approx 0.1 H \tau_{0}\left(\rho_{0} A_{V}\right)^{-1} \approx 40 \mathrm{~cm} \mathrm{sec}^{-1},
$$

which is also reasonably close to observed values and is really not much greater than (5.1).

Estimating, roughly, the magnitude of the vertical velocity as $w \approx E_{V} S^{-\frac{1}{2}}$, we find $w_{d} \approx 2 \tau_{0}\left(\rho_{0} H N\right)^{-1} \approx 10^{-2}$ $\mathrm{cm} \mathrm{sec}{ }^{-1}$, which seems to be a reasonable magnitude for vertical velocities in an upwelling region. Additionally, the onshore velocity has a magnitude $u \approx E_{V}$, which gives $u_{d} \approx \tau_{0} /\left(\rho_{0} H \Omega\right) \approx 1 \mathrm{~cm} \mathrm{sec}^{-1}$. Note that the values 
of the eddy coefficients, which enter in both estimates for $v_{d}$, do not enter in the estimates for $w_{d}$ or $u_{d}$ and that $\left(w_{d} / u_{d}\right) \approx 2 \Omega / N$.

The importance, in the upwelling boundary layer, of the neglected nonlinear terms may be estimated by looking, e.g., at (2.1c) and examining the magnitude of the ratio $R=\epsilon u v_{x} /(2 u) \approx \frac{1}{2} \epsilon \mathrm{O}(v) / \delta_{x}$. [Similar estimates result from the other term $\epsilon w v_{z} /(2 u)$ in (2.1c) and for the terms, e.g., $\epsilon w T_{z} / 4 S w$, in (2.1e).] During the $t_{1}$ scale, $v=\mathrm{O}\left(E_{V^{\frac{1}{3}}}\right)$ and

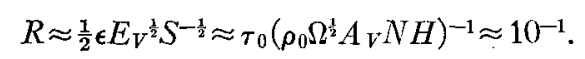

On the $t_{2}$ scale, the maximum value of $v$ is approximately twice as large, from our estimates (5.1)-(5.3); consequently

$$
R \approx 2 \times 10^{-1} \text {. }
$$

It seems, since $R$ is fairly small, that the neglect of the nonlinear terms may be justified within the limits of this model. However, given the approximate nature of order-of-magnitude estimates, $R$ is certainly not small enough to convincingly rule out the possibility that nonlinear effects could be important.

Finally, we point out that the impressed horizontal length scale $L$ has not entered in any of the above estimates. The only requirements on $L$ are that $L \gg L_{x 1}$, $L_{x 2}$, i.e., that $S^{\frac{1}{2}} \ll 1, \lambda^{\frac{1}{2}} \gg 1$.

\section{Summary}

The response, to an impulsively applied alongshore wind stress, of a linear, two-dimensional, $f$-plane model of upwelling in a continuously stratified ocean has been examined. For the stratification parameter $S$ in the range $\mathrm{O}\left(E_{V^{\frac{1}{2}}}\right)<S \lambda \leqslant \mathrm{O}(1)$, an inviscid, baroclinic coastal jet was found to develop initially on a time scale $t_{1}=\mathrm{O}\left(E_{V^{-\frac{1}{2}}}\right)$ in an upwelling boundary layer of thickness $\mathrm{O}\left(S^{\frac{1}{2}}\right)$. The jet adjusted through diffusive processes to a steady state on the longer time scales, $t_{2}=\mathrm{O}\left(S E_{H}^{-1}\right)$ and $t_{3}=\mathrm{O}\left(E_{V}{ }^{-1}\right)$.

Estimates of the parameter values for the upwelling region on the continental shelf off the coast of Oregon show that the dimensional width of the $O\left(S^{\frac{1}{2}}\right)$ upwelling region is $L_{x}=20 \mathrm{~km}$ and that, if it is assumed that $A_{V}=50 \mathrm{~cm}^{2} \mathrm{sec}^{-1}$, the condition $S \lambda>\mathrm{O}\left(E_{V^{\frac{1}{2}}}\right)$ is satisfied if $A_{H} \ll 7 \times 10^{6} \mathrm{~cm}^{2} \mathrm{sec}^{-1}$. The formation of the coastal jet is initiated on the $t_{1}$ time scale, which is the order of several days. The diffusive adjustment of the coastal current to a steady state occurs, however, on a time scale which is comparable with the length of the upwelling season. Estimates for the magnitudes of the velocity components give values which are in reasonable accord with observations.

Fcknowledgments. This research was supported by the Oceanography Section, National Science Foundation, under Grant GA-30592. It is a theoretical con- tribution to CUE (Coastal Upwelling Experiment) which is an IDOE/NSF sponsored project.

\section{APPENDIX \\ Corner Regions}

Fluid upwells into the surface Ekman layer at the coast, $x=1$, through two corner regions with scaling

$$
\begin{array}{ll}
\delta_{x}=\mathrm{O}\left(E_{H^{\frac{1}{2}}}\right), \delta_{z}=\mathrm{O}\left(E_{V^{\frac{1}{2}}}\right) & \text { [region (i) }], \\
\delta_{x}=\mathrm{O}\left(E_{I^{\frac{1}{2}}}\right), \delta_{z}=\mathrm{O}\left[E_{V^{\frac{1}{2}}}(\sigma S \lambda)^{-\frac{1}{2}}\right] & {[\text { region (ii) }] .}
\end{array}
$$

For $S \lambda<O(1)$, corner region (i) is the same, to lowest order, as that in homogeneous models and has been analyzed by Pedlosky (1968). The scaling for region (ii) emerged in the study by Pedlosky (1969) and was given by Blumsack (1972). For $\mathrm{O}\left(E_{V^{\frac{1}{2}}}\right)<S \lambda \leqslant \mathrm{O}(1)$ and $\sigma=\mathrm{O}(1), \delta_{z}$ in this region is in the range $\mathrm{O}\left(E_{V^{\frac{1}{2}}}\right) \leqslant \delta_{z}$ $<\mathrm{O}\left(E_{V^{\frac{1}{6}}}\right)$. We will refer to regions (i) and (ii) as the Ekman layer corner region and the upwelling corner region, respectively.

We analyze below upwelling corner region (ii) in more detail. For that purpose it is convenient not to use correction variables, but to consider the solution to be represented by a separate expansion which is valid in this region. The variables are stretched and expanded as follows:

$$
\begin{aligned}
& \rho=(1-x) E_{H}^{-\frac{1}{2}}, \quad \zeta=(1-z)(\sigma S \lambda)^{\frac{1}{2}} E_{V}{ }^{-\frac{1}{2}}, \\
& u=E_{V^{\frac{1}{2}}}(\sigma S \lambda)^{\frac{1}{2}} \hat{u}(\rho, \zeta, l)+\cdots, \quad v=E_{V^{\frac{1}{2}}}(\sigma S \lambda)^{\frac{1}{2}} \hat{v}+\cdots, \\
& w=\lambda E_{H}{ }^{\frac{1}{2}} \hat{w}+\cdots, \quad p=E_{V^{\frac{1}{2}}}(\sigma S \lambda)^{\frac{1}{2}} E_{H^{\frac{1}{2}}} \hat{p}+\cdots, \\
& T=(\sigma S \lambda) E_{I^{\frac{1}{2}}} \hat{T}+\cdots, \quad \psi=E_{V} \hat{\psi}+\cdots,
\end{aligned}
$$

where

$$
\hat{u}=-\hat{\psi}_{\zeta}, \quad \hat{\imath}=\hat{\psi}_{\rho} .
$$

For $S \lambda=\mathrm{O}(1)$, region (ii) merges with region (i) and all of the terms in (2.2) are important. Only the case $S \lambda<\mathrm{O}(1)$ will be considered here. The equations in region (ii) for $S \lambda<O(1)$ are

$$
\begin{gathered}
\hat{u}_{\rho}+\hat{v}_{\zeta}=0, \\
\hat{u}_{t}-2 \hat{v}=\hat{p}_{\rho}+\hat{u}_{\rho \rho}, \quad \hat{v}_{t}+2 \hat{u}=\hat{v}_{\rho \rho}, \\
\hat{p}_{\zeta}=-\hat{T}, \quad \sigma \hat{T}_{t}+4 \hat{v}=\hat{T}_{\rho \rho} .
\end{gathered}
$$

Eqs. (A2a,b,c) are the same as those in the $E_{H^{\frac{1}{2}}}$ upwelling layer in homogeneous models (Pedlosky, 1968) and this upwelling corner region is evidently the remanent in a stratified fluid of that layer.

It is evident from the scaling in (A2) that the flow in this region adjusts on a short, $t=\mathrm{O}(1)$, time scale. For simplicity, therefore, we consider only the steady-state solution. In the steady state, one equation may be derived for $\hat{\psi}$, i.e.,

$$
\frac{\partial^{6} \hat{\psi}}{\partial \rho^{4} \partial \zeta^{2}}+4 \frac{\partial^{2} \hat{\psi}}{\partial \zeta^{2}}+4 \frac{\partial^{2}}{\partial \rho^{2}}=0 .
$$


The boundary conditions are

$$
\begin{array}{r}
\hat{\psi}(\zeta=0)=-\frac{1}{2} \tau_{T}(x=1)\left[1-e^{-\rho} \cos \rho\right], \\
\hat{\psi}(\rho=0)=0, \quad \hat{\psi}_{\rho \rho \zeta \zeta}(\rho=0)=0, \\
{\left[\hat{\psi}(\rho \rightarrow \infty)+\frac{1}{2} \tau_{T}(x=1)\right] \rightarrow 0, \quad \hat{\psi}(\zeta \rightarrow \infty) \rightarrow 0 .}
\end{array}
$$

Condition (A4a) follows from matching with the solution for the Ekman layer corner region (Pedlosky, 1968), and (A4c) follows from (A2b,d) and the boundary conditions $\hat{v}(\rho=0)=\hat{T}_{\rho}(\rho=0)=0$.

The solution to (A3) with boundary conditions (A4) may be obtained with the aid of a Fourier sine transform in $\rho$ and is

$$
\hat{\psi}=\int_{0}^{\infty} \phi(\zeta, \hat{\lambda}) \sin \rho \hat{\lambda} d \hat{\lambda}
$$

where

$$
\phi(\zeta, \hat{\lambda})=-\frac{4 \tau_{T}(x=1)}{\pi \hat{\lambda}\left(\hat{\lambda}^{4}+4\right)} \exp \left[-2 \hat{\lambda}\left(\hat{\lambda}^{4}+4\right)^{-\frac{1}{2} \zeta}\right] .
$$

For $\zeta \gg 1$, (A5) may be evaluated approximately by Laplace's method; the result is

$$
\hat{\psi} \sim-\tau T(x=1) \pi^{-1} \tan ^{-1}(\rho / \zeta) .
$$

For $\rho \gg 1$, contour integration may be used and Laplace's method applied along the imaginary $\hat{\lambda}$ axis. The behavior is again given by (A6).

The expression (A6) matches, for general values of $\sigma$, with the expansion, for $(1-x) S^{-\frac{1}{2}} \ll 1,(1-z) \ll 1$, of the sum of the steady-state interior (3.23a) and hydrostatic layer streamfunctions $\psi_{0}+\tilde{\psi}_{\mathrm{s}}$. $\left[\tilde{\psi}_{\mathrm{S}}\right.$ is the solution to (4.8) and is given for $\sigma=1$ by (3.25)]. It also matches, for $\sigma=1$, with the expansion of the unsteady solutions on the $t=\mathrm{O}\left(E_{V^{-\frac{1}{2}}}\right)$ time scale [(3.13a) plus either (3.22a) or (3.25)]. It does not match with these unsteady solutions, however, if $\sigma \neq 1$. Therefore, for $\sigma \neq 1$, there must be a further adjustment, on the $t=\mathrm{O}\left(E_{V}{ }^{-\frac{1}{2}}\right)$ time scale, between the solution in the upwelling corner region (ii) and that in the inviscid $\delta_{x}=\mathrm{O}\left(S^{2}\right)$ layer. This adjustment evidently takes place in a region which is growing with time and which eventually becomes, on the $t=\mathrm{O}\left(S E_{H}{ }^{-1}\right)$ time scale, the boundary layer described in Section 4. The approximate governing equations for this region, for $S \lambda<\mathrm{O}(1)$, are

$$
\begin{gathered}
u_{x}+w_{z}=0, \\
2 v=p_{x}, \quad v_{i}+2 u=E_{H I} v_{x x}, \\
T=p_{z}, \quad T_{t}+4 S w=\left(E_{I I} / \sigma\right) T_{x x} .
\end{gathered}
$$

Eqs. (A7) are included in (A2) and are the same, for $S \lambda<O(1)$, as (4.4). The scales for $x$ and $z$, as a function of $t$, may be found from (A7):

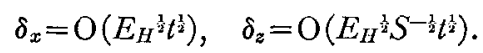

For $t=\mathrm{O}(1)$, (A8) gives the same magnitudes for $\delta_{x}$ and $\delta_{z}$ as in (Alb). For $t=\mathrm{O}\left(E_{V}{ }^{-\frac{1}{2}}\right)$, we find

$$
\delta_{x}=\mathrm{O}\left(E_{H^{\frac{1}{2}}} E_{V}{ }^{-\frac{1}{4}}\right), \quad \delta_{z}=\mathrm{O}\left[E_{V^{\frac{1}{4}}}(S \lambda)^{-\frac{1}{2}}\right]
$$

and for $t=\mathrm{O}\left(S E_{H}{ }^{-1}\right)$ we obtain, as expected, the scales in Section 4, i.e.,

$$
\delta_{x}=\mathrm{O}\left(S^{\frac{1}{2}}\right), \quad \delta_{z}=\mathrm{O}(1) .
$$

We do not attempt to solve (A7) on the $t=\mathrm{O}\left(E_{V}{ }^{-\frac{1}{2}}\right)$ time scale and to link, for $\sigma \neq 1$, the asymptotic form of the upwelling corner region solution (A6) with the solutions (3.22a) and (3.25) for the inviscid $\delta_{x}=\mathrm{O}\left(S^{\frac{1}{2}}\right)$ layer. We note, however, that the solution to (A7) should provide this joining since (A7) contains both $\mathrm{Eq}$. (3.16) for the inviscid $\delta_{x}=\mathrm{O}\left(S^{\frac{1}{2}}\right)$ layer and, in the steady state, Eq. (4.8) whose solution does match (A6).

\section{REFERENCES}

Allen, J. S., 1971: Some aspects of the initial value problem for the inviscid motion of a contained, rotating, weakly stratified fluid. J. Fluid Mech., 46, 1-23.

, 1972: Upwelling of a stratified fluid in a rotating annulus: Steady state. Part 1. Linear theory. J. Fluid Mech., 56, 429-445.

Barcilon, V., and J. Pedlosky, 1967: A unified linear theory of homogeneous and stratified rotating fluids. J. Fluid Mech, $29,609-621$.

Blumsack, S. L., 1972: The transverse circulation near a coast. $J$. Phys. Oceanogr., 2, 34-40.

Carrier, G. F., 1965: Some effects of stratification and geometry in rotating fluids. J. Fluid Mech., 23, 145-172.

Charney, J. G., 1955: The generation of oceanic currents by wind. $J$. Marine Res., 14, 477-498.

Csanady, G. T., 1968: Motions in a model great lake due to a suddenly imposed wind. $J$. Geophys. Res., 73, 6435-6447.

Durance, J. A., and J. A. Johnson, 1970: East coast ocean currents. J. Fluid Mech., 44, 161-172.

Fofonoff, N. P., 1962 : Dynamics of ocean currents. The Sea, Vol. 1, New York, Interscience, 323-395.

Garvine, R. W., 1971: A simple model of coastal upwelling dynamics. J. Phys. Oceanogr., 1, 169-179.

Greenspan, H. P., 1968: The Theory of Rotating Fluids. Cambridge University Press, $327 \mathrm{pp}$.

Holton, J. R., 1965: The influence of viscous boundary layers on transient motions in a stratified rotating fluid. Parts 1 and 2. J. Almos. Sci., 22, 402-411; 535-540.

Hurlburt, H. E., and J. D. Thompson, 1973: Coastal upwelling on a $\beta$-plane. J. Phys. Oceanogr., 3, 16-32.

Hsueh, Y., and R. N. Kenney, 1972: Steady coastal upwelling in a continuously stratified ocean. J. Phys. Oceanogr., 2, 27-33.

Mooers, C. N. K., C. A. Collins and R. L. Smith, 1973: The dynamic structure of the frontal zone in the coastal upwelling region off Oregon. (Submitted to J. Phys. Oceanogr.)

O'Brien, J. J., and H. E. Hurlburt, 1972: A numerical model of coastal upwelling. J. Phys. Oceanogr., 2, 14-20.

Pedlosky, J., 1967: The spin-up of a stratified fluid. J. Fluid Mech., 28, 463-479.

-, 1968: An overlooked aspect of the wind-driven oceanic circulation. J. Fluid Mech., 32, 809-821.

- 1969: Linear theory of the circulation of a stratified ocean. J. Fluid Mech., 35, 185-205.

Siegmann, W. L., 1971: The spin-down of rotating stratified fluids. J. Fluid Mech., 47, 689-712.

Walin, G., 1972: On the hydrographic response to transient meteorological disturbances. Tellus, 24, 169-186. 